\title{
Die Auflösung des Klosters Buch bei Leisnig während der Reformation
}

\author{
Michael Beyer
}

Alle wichtigen Ereignisse aus den Anfangsjahren der Reformation in Leisnig sind im Beitrag von Heiko Jadatz bereits angeklungen: Der Streit um die Pfarrstelle. Die hinhaltende, das alte System nicht schützende Verhandlungstaktik Kurfürst Friedrichs des Weisen, die auch der evangelischen Bewegung in Leisnig Vorschub leistete. Schließlich der direkte landesherrliche Eingriff in die Wahl eines neuen Abtes 1526 und die bald darauf einsetzende landesherrlich gesteuerte Auflösung bzw. Aufteilung des gesamten, nicht eben kleinen Klosterbesitzes.

Die „Säkularisierung“ des Klosters Buch begann jedoch mit der Entscheidung der Leisniger, sich einen eigenen Pfarrer zu wählen. Denn das war ein schwerer Eingriff in die Rechte des Klosters an der Parochie Leisnig, deren Einkünfte und Pfarrrechte vollständig in das Kloster inkorporiert waren. Auch wenn die Kastenordnung in Leisnig selbst in den ersten Jahren nicht optimal funktionierte, so hatte Leisnig mit ihr seinen mittelalterlichen Parochialstatus doch verändert, und zwar auf Dauer. Insofern wurde sie zum Vorbild der Neuordnung von Kirchengut in kommunalen Zusammenhängen und darüber hinaus. Natürlich gab es eine formale Kontinuität: die Parochie bestand weiter, allerdings, und das ist entscheidend, mit einer klaren evangelischen Grundierung, und bald als Teil des sich entwickelnden reformatorischen Kirchenwesens in Sachsen.

Das Ende der „Säkularisierung“ Buchs genau zu bestimmen, ist ungleich schwieriger. Die mittelalterliche, geistliche Institution „Kloster“ hörte spätestens während der Visitation von 1534 auf zu existieren. Das geschah durch die Verordnung der Visitationskommission, die Stundengebete der Mönche einzustellen, sie der evangelischen Predigt zu unterwerfen und Austrittswillige abzufinden. Das Kloster als Grundherr hatte bereits 1526 seine Existenz verloren, nämlich als sein gesamter Besitz und alle damit im Zusammenhang stehenden Gerechtsame unter kurfürstliche Verwaltung gestellt wurden. So gesehen war elf Jahre nach der Initiative der Leisniger das Kloster „säkularisiert“. Das scheint ein langer Zeitraum zu sein, gerade im Vergleich mit anderen Territorien und Städten im Reich. Aber dieser lange Zeitraum hatte eine einfache Ursache: Die Reformationsgeschichte Kursachsen ist seit ihrem Beginn - Stichworte „Wildwuchs“ und „Kultivierung“ - die Geschichte einer Suchbewegung, innerhalb derer sich neue Formen des Kirchenwesens vor Ort ausbilden, was zunächst unter einer besonderen Bedingung, nämlich der Duldung durch die Landesherrschaft, abläuft. Dieser Duldung folgt seit 1526 eine sich fortwährend verdichtende Institutionalisierung hin zu einem landesherrlich kontrollierten Kirchenwesen, die allerdings selbst noch für längere Zeit als Suchbewegung bezeichnet werden kann. Dazu gehört in erster Linie die Herausbildung der landesherrlichen Visitation, die theoretisch wie praktisch als geistlich-weltliche Institution entwickelt wird und unter der persönlichen Verantwortung des christlichen Landesherrn als eines „Notbischofs" steht.

Eine detaillierte Auflistung der Veränderungen all der größeren und kleineren Besitz- und Rechtstitel des Klosters Buch kann hier nicht erfolgen. Das wäre aufgrund der bereits bearbeiteten Quellenüberlieferung gar nicht möglich. Die moderne Literatur zur genauen Besitzgeschichte des Klosters endet bisher regelmäßig mit der Reformation. Abhilfe wird hier in absehbarer Zeit das in Vorbereitung befindliche „Sächsische Klosterbuch“ schaffen, für das Alexander Sembdner den einschlägigen Artikel verfasst hat. Zunächst ist zu klären, um welchen Vorgang es sich bei der „Säkularisierung“ jenseits der formalen Zuordnungen gehandelt hat. Wir geraten damit in die nicht eben leichte Materie des Umgangs mit dem mittelalterlichen Kirchengut während der Reformation. Der Begriff „Säkularisation" eignet sich nur unzureichend, auch wenn er immer wieder bemüht wird. In Anführungszeichen gesetzt, mag er auch weiterhin dienen, aber nur als Signal, ihn für die Reformationszeit genauer bestimmen zu müssen.

Die Vorgänge um das Kloster als geistlicher Gemeinschaft und um seinen weltlichen Besitz können weitgehend als exemplarisch für den Umgang mit einem großen Teil des Kirchenguts in Sachsen im Reformationsjahrhundert angesehen werden. Die Besonderheit Buchs liegt in seiner Einbindung in die Leisniger Reformationsgeschichte, die wiederum durch die Kasten- 


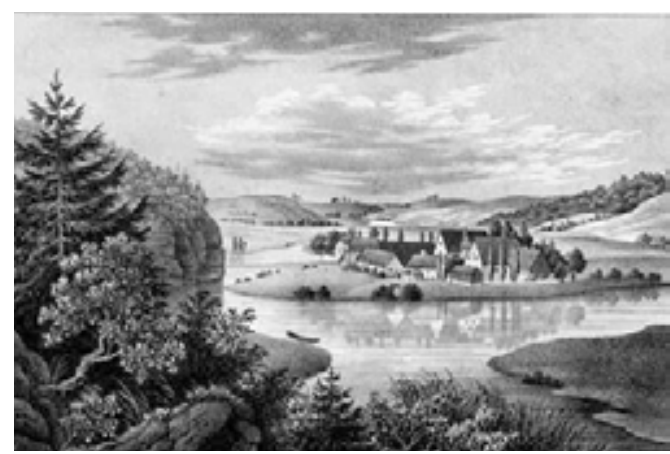

ordnung und Luthers direkten Einfluss exemplarischen Charakter hatte.

\section{„Säkularisierung“ und Kirchengut}

Der Begriff „Säkularisierung“ steht heute ganz allgemein für Übergänge von materiellen Gütern, aber auch von Phänomenen des Denkens heraus aus kirchlichen und hinein in weltliche Zusammenhänge. Im engeren Sinn bezeichnet man damit den Übergang von Kirchengütern durch den Staat sowie auch die kulturelle Loslösung aus kirchlicher Vormundschaft. Im kirchenrechtlichen Sprachgebrauch gibt es dafür den lateinischen Begriff „saecularizatio“, der sowohl für das gewaltsame Enteignen sogenannter „geistlicher Güter“ als auch für die Laisierung von Priestern und Mönchen gebraucht wird. Im 16. Jahrhundert existierte dieser Begriffsgebrauch noch nicht. „Secularis“ meint damals einfach allgemeine Zuordnungen zur weltlichen Sphäre. In der modernen Bedeutung taucht der Begriff erst im 17. Jahrhundert im Zusammenhang mit dem Westfälischen Frieden auf.

Gebraucht man „Säkularisierung“ als historischen Begriff für die Verweltlichung des Kirchengutes, steht man vor dem Problem, das dieses im 16. Jahrhundert nicht einfach „verweltlicht" wurde. Es kam zwar weithin unter weltliche, d. h. landesherrliche bzw. kommunale Aufsicht, wurde aber großenteils seinem traditionellen kirchlichen Verwendungszweck zugeführt (Kirche, Schule, Fürsorge). Dem wurde im staatskirchenrechtlichen Sprachgebrauch Rechnung getragen und der Begriff „,Reformation' des Kirchengutes“ eingeführt. Im kirchenhistorischen Zusammenhang spricht man von der „Neuordnung des Kirchengutes“. Letztere Begrifflichkeit ergibt sich aus einer theologischen Problematisierung, die Luther vornahm, als er konsequent zwischen geistlichen und weltlichen Gütern unterschied. Der Begriff „geistliches Gut“ konnten niemals materielles Gut sein, auch wenn damit traditionell das Kirchengut - nicht zuletzt aus Gründen der Bestandssicherung - bezeichnet wurde. In der 62 . seiner 95 Thesen bestimmte er z. B. „das wahre Gut der Kirche [... als] das heilige Evangelium von der Herrlichkeit und Gnade Gottes“. Die Predigt dieses Evangeliums schafft den Glauben, dem Christen durch gute Werke in der Gemeinde Gestalt geben. Luther hat das sehr eindrücklich in einen engen Zusammenhang mit dem Gottesdienst der Gemeinde gebracht. Hier, wo das Evangelium hörbar wird, kommt es in der Folge durch eine Vielzahl guter Werke zur Sammlung weltlicher Güter, die wiederum dem Gottesdienst zugewendet werden, wobei „Gottesdienst" durch die bereits genannte Trias von Kirche, (Hoch-)Schule und (Armen-)Fürsorge beschrieben werden kann.

\section{Leisniger Konkretionen}

Wie aber kommt eine christliche Gemeinde auf legitime Weise zu einem solchen Gottesdienst? Luther unterschied auch im Rechtsbereich streng zwischen geistlichen und weltlichen Rechten der Gemeinde: Im geistlichen Bereich hatte sie alles Recht auf ihrer Seite, konnte z. B. also die geistliche Seite der Inkorporation der Leisniger Pfarrstelle in das Kloster Buch kraft evangelisch verstandenen geistlichen Rechts unterbinden und die Pfarrstelle personell selbst besetzen. Das überkommene Kirchengut rechnete nach Luther unter die weltlichen Angelegenheiten der Kirche. Besitztitel aber mussten dem weltlichen Recht unterstellt bleiben, weshalb die Gemeinde die Inkorporation zum Kloster Buch nicht vollständig lösen konnte und nun vor der Aufgabe stand, in Übereinstimmung mit dem christlichen Gottesdienst eigenes Kirchengut zu generieren. Ein Argument für die Weiterverwendung alten Kirchenguts, das schon in Leisnig gebraucht wurde und in der Folgezeit große Bedeutung bekommen sollte, war die Übereinstimmung seiner Verwendung mit dem ursprünglichen, nun im evangelischen Sinn gedeuteten Willen der ehemaligen Stifter von Kirchengut. Diese komplizierte Ordnungsaufgabe zwischen geistlichen und weltlichen Gütern, die sowohl die christliche Gemeinde als auch die weltliche Obrigkeit zu lösen hatte, wurde 1523 in Leisnig geleistet, wobei Luther selbst durch Leisniger Christen zur Mitgestaltung aufgefordert worden war. Luther hat jegliches Streben nach festen Ordnungen für christliche Gemeinden immer mit einem gewissen Misstrauen beobachtet. Er scheute den Rückfall in die gerade überwundenen Zustände in der römischen Kirche, in der das Kirchenrecht als gewichtiger Teil kirchlicher Tradition praktisch oft die Bibel hintangestellt hatte. Im Fall von Leisnig scheint er den Ordnungswillen akzeptiert zu haben. Das mag
Kloster Buch an der Freiberger Mulde, Lithographie um 1840 aus: Sachsens Kirchengalerie 
daran liegen, dass Anfang 1522 in Wittenberg selbst geistliche Elemente in eine Stadtordnung eingefügt werden sollten, was Luther bewog, die Wartburg zu verlassen, um die Vermischung von geistlichen und weltlichen Aufgaben zu unterbinden. Hier in Leisnig konnte er das Unterscheiden der beiden Bereiche und ihr Miteinander selbst mitgestalten. Augenfällig wird dies dadurch, dass Luther für Leisnig gleich drei Schriften veröffentlichte. Für die Auflösung der geistlichen Inkorporation der Pfarrstelle lieferte er eine Begründung der freien Pfarrwahl der Gemeinde. Daneben erstellte er für Leisnig eine eigene Gottesdienstordnung und verfasste $\mathrm{zu}^{-}$ dem ein ausführliches Vorwort zur „Ordnung eines Gemeinen Kastens“, in dem er sich grundsätzlich zum Umgang mit Kirchengut auseinandersetzte, von dem er mit Recht annahm, dass vor allem durch die Selbstauflösung von Klöstern große Mengen davon in naher Zukunft zur Disposition stehen würde.

Als grundlegender Baustein der Leisniger Reformation muss die Kastenordnung angesehen werden, weil mit ihr die finanzielle Basis für eine unabhängige Kirchgemeinde gelegt wurde. Ihre Voraussetzung bestand in einem Vertrag zwischen drei für Leisnig bestimmende Gruppen: dem städtischen Rat, dem ansässigen Adel und Vertretern der Handwerke. Letztere besiegelten den Vertrag auch für die ganze Einwohnerschaft und die nach Leisnig eingepfarrten Bauern umliegender Dörfer. Der Vertrag trug den Namen „Brüderliche Vereinigung“, ein Begriff, der sich zuvor in süddeutschen ritterschaftlichen Zusammenhängen und später im täuferischen Bereich findet. Hier in Leisnig scheint „Brüderliche Vereinigung“ in Verbindung zu stehen mit einem erneuerten Verständnis der spätmittelalterlichen Bruderschaften, wie es Luther 1519 ihren Mitgliedern in einer kleinen Schrift nahegelegt hatte. Wollte eine solche Gruppe innerhalb einer Stadt etwas wirklich Christliches unternehmen und ihre Finanzen nicht nur für sich selbst ausgeben, dann sollten sie eine gemeinsame Kasse schaffen, die speziell auf die Finanzierung de Armenpflege ausgerichtet sein sollte. Auf dieser Grundlage dürfte bereits der Wittenberg Gemeine Kasten geschaffen worden sein. Hier in Leisnig ist der der „Brüderlichen Vereinigung“ zugrundeliegende reformatorische Bruderschaftsgedanke als Synonym für die Trägerschaft aller Festlegungen der Kastenordnung mit ihrer Hinordnung auf Gottes Ehre und der Liebe zum Nächsten im Sinne des Gemeinnutzes zu verstehen. Alle Beteiligten verpflichteten sich somit, die finanziellen Grundlagen für das Pfarramt, d.h. die Predigt von Gottes Wort, sowie für das äußere christliche Leben der sich nun als Kirch- gemeinde verstehenden Glieder der Parochie Leisnig zu schaffen.

Um es nochmals zu betonen: In der Kastenordnung ging ganz deutlich nicht um konkrete geistliche Aufgaben, sondern allein um weltliches Gut in seiner Verbindung zum Gottesdienst der Gemeinde, und dessen willen sich die Beteiligten zuvor in einer besonderen Art von Verein, als Kirchgemeinde, konstituiert haben. Der Zugriff zum gemeinsamen Kirchengut konnte nur gemeinsam erfolgen - jede der miteinander „brüderlich vereinigten“ Gruppen (die Bauernschaft wird hier gesondert beteiligt) verfügte über jeweils einen der vier Schlüssel zu den vier Schlössern des Kastens, wobei der Pfarrer als der geistlich Schlüsselgewaltige keinen eigenen Schlüssel zum weltlichen Gut der Gemeinde besaß. Die Hinordnung des gemeinsamen Gutes zum Gottesdienst im oben beschriebenen umfassenden Sinn wurde jedoch durch den Aufstellungsort des Kastens, die Kirche, klar herausgestellt.

Luther stellte in seiner Vorrede zur Kastenordnung eigens noch einmal zwei wichtige Elemente heraus: Die Leisniger hätten „eine neue Ordnung für den Gottesdienst und ein „Gemeindeeigentum nach dem Vorbild der Apostel“ beschlossen. Ich halte letzte Bemerkung im Zusammenhang mit dem Begriff der „brüderlichen Vereinigung“ für den Versuch einer neuen, evangelisch-kirchenrechtlich sauberen Konstitution der evangelischen Gemeinde überhaupt. Wenn Luther auch vorher oft von "Gemeinde“ sprach, handelte es sich $\mathrm{m}$. E. nicht um die herkömmliche Parochialgemeinde, sondern um „Gemeinde“, wie sie seiner Meinung nach sein sollte, eben Gemeinde nach dem Vorbild der Apostel, deren Glieder der allgemeinen geistlichen Kirche angehören, Leib Christi sind. Weil aber Christen in der Welt als sichtbare Gemeinde situiert sind, muss diese Gemeinde notgedrungen Institutionen ausbilden, die der Unterscheidung nach ihrem geistlichen und weltlichen Aspekt entsprechen. Die „brüderliche Vereinigung“ der Christen war in Leisnig deckungsgleich mit den Mitgliedern der Kommune. Aber indem diese sich als brüderliche Vereinigung konstituierte, man könnte sie auch als Bruderschaft oder christlichen Verein bezeichnen, begründete sie zugleich als ihre materielle Basis eine eigene weltliche Institution - den gemeinen Kasten, worunter eine allgemeine Kirchkasse zu verstehen ist. In dieser Konstellation ist das Verhältnis von Gottesdienst (geistlicher Aspekt) und Kirchengut (weltlicher Aspekt) gewahrt. Bei der Kastenordnung handelte sich nicht eigentlich um eine besondere neue Sozialordnung, denn sie lehnte sich an ältere Vorbilder an. Und 
dennoch war sie etwas Besonderes: Denn es handelte sich um eine reformatorisch-zeitgemäße Sozialgestalt der Kirche, in der Glaube und Liebe nicht zu trennen waren. Mit der Leisniger Kastenordnung trat also die materielle, weltlichrechtlich geordnete Basis der geistlich freien Kirchgemeinde ins Leben.

Deshalb muss es auch nicht verwundern, dass Luthers Vorwort zur Kastenordnung einen Titel trägt, der bereits auf übergemeindliche Zusammenhänge hinweist: „Vorschlag, wie mit den „geistlichen“ [d. h. weltlichen] Gütern [der Kirche] umzugehen ist“. Leisnigs Beispiel möge zum Untergang der Klöster und vieler Institutionen der alten Kirchenorganisation führen. Aber zugleich musste klar sein: „einige habgierige Wänste“ dürften sich nicht am dabei anfallenden alten Kirchengut bereichern. Mönche und Nonnen, die freiwillig ihre Klöster verlassen, sollten durch die Teilrückgabe ihres eingebrachten Gutes abgefunden werden; Vereinbarungen zwischen der weltlichen Obrigkeit und den in ihrem Einflussbereich befindlichen Klöster sollten keine Neuaufnahmen gestatten, aber die obrigkeitliche Verwaltung musste die lebenslange, auskömmliche Versorgung der Bleibenden gewährleisten. Luther dachte hier auch über einen landesweiten „gemeinen Kasten“ im Dienst der allgemeinen Armenfürsorge nach und in parallelen Äußerungen in dieser Zeit über die Umwandlung der bischöflichen Territorien in weltliche Herrschaften, über Wucherausgleich und über Schulgründungen für Mädchen und Jungen in ehemaligen Bettelordensklöstern. Insgesamt schwebte ihm eine differenzierte Neuordnung des alten Kirchengutes unter Berücksichtigung der allgemeinen Armut vor. Die Umstände der „Säkularisierung“ des Klosters Buch brachten also nicht nur lokale Veränderungen, sondern wurden zum Ausgangspunkt entscheidender reformatorischer Überlegungen und Handlungen.

\section{Zur Neuordnung des Kirchenguts aus dem Besitz des Kloster Buch}

Der umfangreiche Besitz des Klosters konzentrierte sich um 1500 auf drei bzw. vier Kerngebiete: das Zentrum um das Kloster selbst, Besitzungen um Altenburg, die Stadt Belgern sowie Besitzungen um Colditz. Die Größe des allein um das Kloster liegenden Besitzes wird deutlich, wenn man den grundherrschaftlichen Umfang des Amtes Leisnig um 1550 betrachtet: mehr als die Hälfte davon gehörte ehemals zum Kloster. In reformatorischer Zeit wurde Buch wie alle Klöster im Land behandelt: Die Neuordnung vor Ort wurde durch die Visitation festgelegt: Abfin-

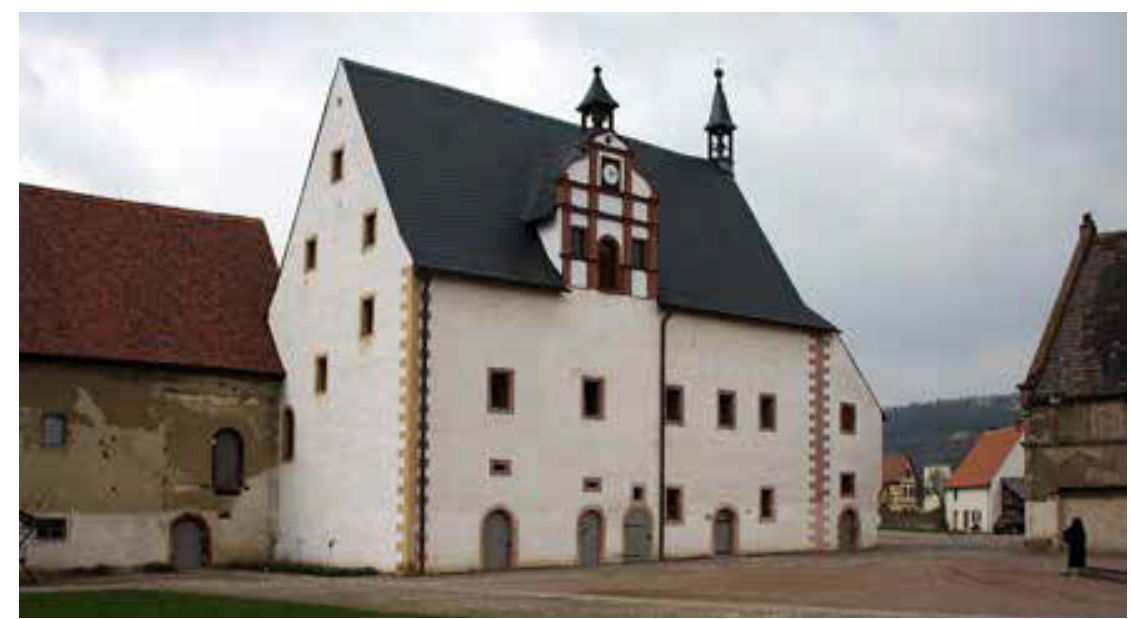

dungen für ehemaliger Mönche erfolgten durch kleine Besitzteile im Umland des Klosters. Die Verwaltung des gesamten Besitzes oblag zunächst kurfürstlichen Beamten. Dabei kam es zur Veräußerung einzelner Liegenschaften sowie zur Sicherung, Verwahrung und Verwertung der überflüssigen Kleinodien zur Edelmetallgewinnung. Der Gesamtbesitz blieb dabei allerdings erhalten. Während der folgenden Sequestration des Klosterbesitzes, d. h. seiner zeitweilig landständischen Verwaltung, wurde ein sehr großer Teil der erzielten Einnahmen zugunsten der Kosten der Neuordnung des Kirchenwesens (landeskirchlicher Ausgleich) und für die Verwaltung selbst aufgewendet. Allgemeine Überzeugung war es, dass Kirchengut für den Fall, dass das Kirchenwesen insgesamt gut ausgestattet war, auch der „allgemeinen Landesnotdurft“ nützlich sein sollte. Ein solcher Fall trat während des Schmalkaldischen Krieges ein: 1548 wurden einzelne Klosterdörfer von Buch beauflagt, zusammen vier Heerwagen und 43 Mann Fußvolk zu stellen. In den folgenden Jahrhunderten wechselte das Klostergut häufig den

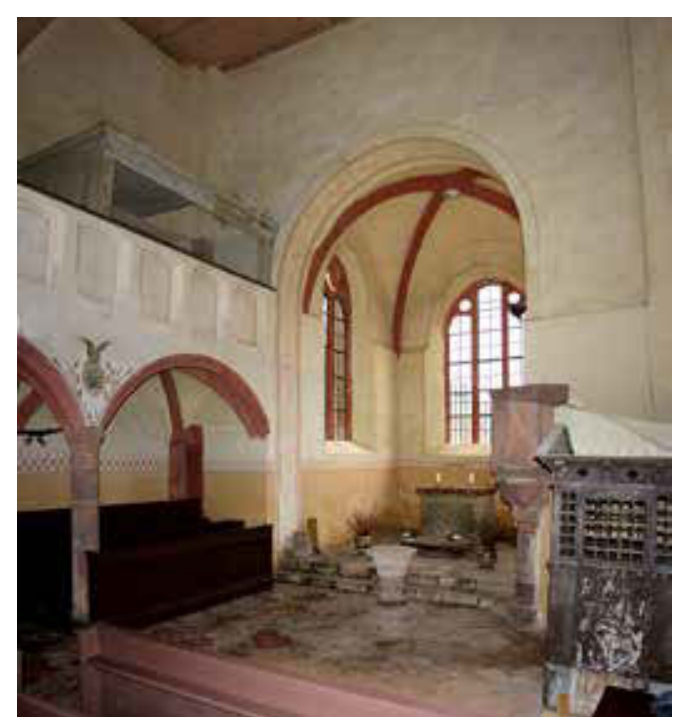

Kloster Buch, Ostteile der ehemaligen Klosterkirche, Ende des 16. Jahrhunderts umgebaut zur Gutskapelle Foto: Matthias Donath
Kloster Buch, Innenraum der Gutskapelle Foto: Matthias Donath 


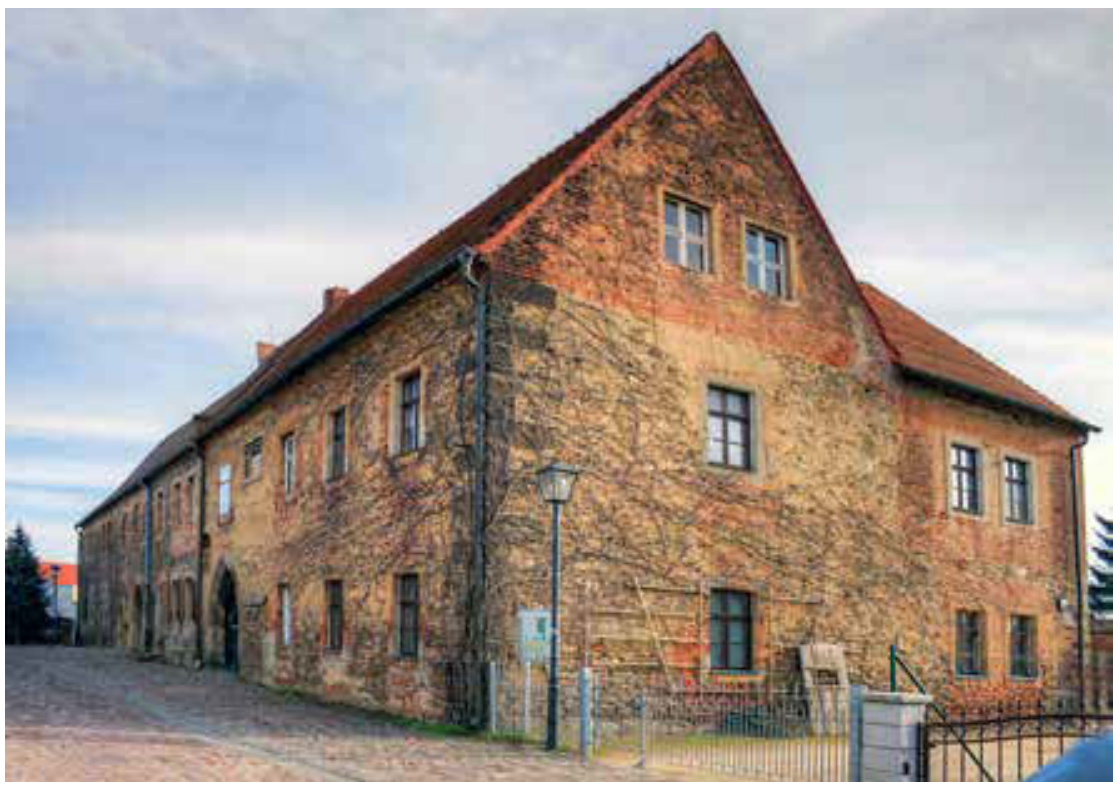

Belgern, ehemaliger Klosterhof des Klosters Buch, heute Pfarrhaus ๑ Wikimedia (Radler59)

Literatur

Herta Battré: Beiträge zur Geschichte des Klosters Buch. Diss. Universität Leipzig 1951. Michael Beyer: Martin Luther und das Kirchengut. Diss. Universität Leipzig 1984.

Thomas Ludwig: Besitzgeschichte des Zisterzienserklosters Buch bei Leisnig. Magisterarbeit Universität Leipzig, Historisches Seminar, Leipzig 1996.

Johann Christian Schlewitz/Johann George Schlewitz: Nachrichten vom Nahmen, Ursprung, Veränderung und verschiedenen Zufällen der Stadt Belgern im Meißner-Lande. Wittenberg 1748.

Alexander Sembdner: Art. Buch, Zisterzienser (OCist), in: Enno Bünz/Sabine Zinsmeyer/ Dirk Martin Mütze (Hrsg.): Die mittelalterlichen Klöster, Stifte und Kommenden im Gebiet des Freistaates Sachsen (in Druckvorbereitung).

Autor

Dr. Michael Beyer Universität Leipzig

Theologische Fakultät Lehrstuhl für Spätmittelalter, Reformation und territoriale Kichengeschichte

Martin-Luther-Ring 3 04109 Leipzig bzw. die Besitzer und wurde dabei immer weiter vermindert. Die Landesherrschaft legte erlöstes Geld teilweise für Zinsen an, aus denen die Fürsten- bzw. Landesschule St. Augustin zu Grimma unterhalten wurde. Der Besitz um den alten Klosterort wurde ein Rittergut, das nach der Bodenreform in SED-Besitz kam und schließlich bis zum Ende der DDR ein Volksgut war.

In Streitigkeiten zwischen dem Abt von Buch und den Bürgern der Kloster-Stadt Belgern griff 1519 die Landesherrschaft ein, um sie zu schlichten. Luther soll auf Bitten von Bürgern 1522 einen Prediger nach Belgern vermittelt haben, der zwar nur acht Tage am Ort blieb, aber großen Zulauf hatte, worauf Abt Anthonius Dytz einen entrüsteten Brief an Rat und Gemeinde schrieb. Bald darauf forderte die Stadt einen neuen Pfarrer, aber es ist nicht überliefert, wie darüber von den kurfürstlichen Räten, die sowohl Vertreter der Bürger als auch den Abt von Buch $\mathrm{zu}$ einer Verhandlung geladen hatten, entschieden wurde. Wahrscheinlich wurde ein Kompromiss geschlossen, denn aus einem Brief Luthers von 1527 an den Kurfürsten geht hervor, dass der evangelische Pfarrer sich in Belgern mühsam durchschlug, während ein Mönch aus Buch noch immer die Pfarrgüter innehatte. Letzteres ist eine Nachricht aus dem Beginn bzw. unmittelbar vor der ersten Visitation. In Belgern herrschten also ähnliche Verhältnisse wie seinerzeit in Leisnig. Wegen der Beachtung der Rechtslage war der Gemeinde der Zugriff auf das inkorporierte Pfründenvermögen der Belgerner Parochie verwehrt, während die geistliche Inkorporation beseitigt war. Im Unterschied zu Leisnig war man in Belgern anscheinend nicht zu einer auskömmlichen Bestellung des evangelischen Pfarramtes gelangt.
Der Klosterhof zu Belgern diente seit der Mitte des 15. Jahrhunderts u. a. als Lateinschule und neben der Schule im Kloster Buch selbst auch als Ordensstudium für die Bucher Zisterzienser. Die Absolventen wurden im Leipziger Bernhardinerkolleg auf ihre Eignung zum Theologiestudium geprüft und hatten im Eignungsfall eine Art Anwartschaft auf Stellen in der Leipziger Philosophischen und Theologischen Fakultät. Der Klosterhof wurde während der Sequestration an Adlige vergeben; um 1570 ging er an die Stadt Belgern, die das Gebäude zur Wohnung für die Pfarrer bestimmte. Es wird bis heute als Pfarramt mit Pfarrwohnung und Archiv sowie mit Räumen für die Gemeinde genutzt. Belgern als ehemaliger Klosterbesitz und mittlerweile kurfürstliche Stadt spielte übrigens auch noch einmal eine Rolle als es in der zweiten Hälfte des 16. Jahrhunderts zum Besitzaustausch zwischen dem Kurfürsten und dem letzten Bischof vom Meißen kam. Eine Station dieses Austauschs - er sollte darauf hinauslaufen, die reichsunmittelbaren Anteile des bischöflichen Besitzes unter kurfürstliche Gewalt zu bringen - war der Tausch des bischöflichen Stolpener Besitzes, der an den Kurfürst ging, und des Amts Mühlberg sowie der Stadt Belgern, die der Bischof bekam.

In Klosterbuch selbst steht neben dem Abtshaus und einigen Klausurgebäuden, die jahrhundertelang als Stallungen genutzt wurden und inzwischen von einem Förderverein museal genutzt werden, die im 17. Jahrhundert aus Vierung und Chor der ehemaligen Klosterbasilika entstandene „Rittergutskirche“. Gegenwärtig wird sie regelmäßig auch gottesdienstlich genutzt. Das Gebäude ist nicht mit einem Besitztitel der Kirche verknüpft, steht aber nach wie vor im Dienst der Verkündigung. Insofern handelt es sich um eine „Säkularisierung“ und ist doch keine. In der Reformationszeit ist mit dem Kirchengut so verfahren worden, dass es in großen Teilen einer Neuordnung unterworfen und in erheblichem Umfang in den Dienst der Verkündigung und der Armenfürsorge auf der Ebene der Einzelgemeinden sowie der Bildung gestellt wurde. In diesen Prozess gehört auch die Geschichte des Klostergutes Buch, nicht zuletzt durch seine jahrhundertelange Beziehung zu St. Augustin in Grimma. Die heutigen Staatsleistungen an die Kirche gehen zwar formal auf die große Säkularisierung geistlicher Territorien zu Beginn des 19. Jahrhundert zurück, müssen aber auch als eine Spätwirkung jener reformatorisch begonnenen Neuordnung verstanden werden, in der die Landesherrschaft einerseits das Kirchengut neu ordnete, andererseits erhebliche Gewinne aus ihm zog. 